\title{
Correction to: Analysis of journal evaluation indicators: an experimental study based on unsupervised Laplacian
} score

\author{
Lin Feng ${ }^{1} \cdot$ Jian Zhou ${ }^{1}$ (D) Sheng-Lan Liu ${ }^{1} \cdot$ Ning Cai $^{2,4}$ (D) Jie Yang $^{3}$
}

Published online: 3 June 2020

๑) Akadémiai Kiadó, Budapest, Hungary 2020

\section{Correction to: Scientometrics} https://doi.org/10.1007/s11192-020-03422-8

The original publication of the article contained error in Table 6 in the header row. The correct table is given below:

The original article can be found online at https://doi.org/10.1007/s11192-020-03422-8.

Jian Zhou

zhoujian@mail.dlut.edu.cn

$\triangle$ Ning Cai

caining91@tsinghua.org.cn

Lin Feng

fenglin@dlut.edu.cn

Sheng-Lan Liu

lius1@dlut.edu.cn

Jie Yang

totoroyang@tsinghua.edu.cn

1 School of Computer Science and Technology, Dalian University of Technology, Dalian 116024, China

2 School of Automation, Beijing University of Posts and Telecommunications, Beijing 100876, China

3 Research Institute of Information Technology, Tsinghua University, Beijing 100084, China

4 Key Laboratory of China's Ethnic Languages and Information Technology for Ministry of Education, Northwest Minzu University, Lanzhou 730030, China 
Table 6 Laplacian scores, class correlations, and accuracy of $k$-NN classification of each feature in class 2

\begin{tabular}{|c|c|c|c|c|c|c|c|c|}
\hline \multirow[t]{2}{*}{ No. } & \multirow[t]{2}{*}{ Feature } & \multirow[t]{2}{*}{ Laplacian score } & \multirow[t]{2}{*}{ Class correlation } & \multicolumn{5}{|c|}{ Accuracy of $k$-NN classification } \\
\hline & & & & $k=1$ & $k=2$ & $k=3$ & $k=4$ & $k=5$ \\
\hline 1 & $\mathrm{JIF}$ & 0.9572 & -0.6533 & 0.5912 & 0.5955 & 0.6152 & 0.6002 & 0.6184 \\
\hline 2 & 5-Year JIF & 0.9186 & -0.5222 & 0.4295 & 0.4263 & 0.4184 & 0.4178 & 0.4163 \\
\hline 3 & CiteScore & 0.9502 & -0.6494 & 0.5245 & 0.5259 & 0.5033 & 0.5184 & 0.5184 \\
\hline 4 & SJR & 0.7728 & -0.5008 & 0.3000 & 0.3160 & 0.3155 & 0.3178 & 0.3133 \\
\hline 5 & SNIP & 0.6980 & -0.4969 & 0.3225 & 0.3233 & 0.3108 & 0.3100 & 0.3126 \\
\hline 6 & H-index & 0.9775 & -0.6304 & 0.5955 & 0.5984 & 0.5678 & 0.5879 & 0.5848 \\
\hline
\end{tabular}

Bold indicates the best performance

The original article has been corrected. 